\title{
On Two-Dimensional Variable Viscosity Fluid Motion with Body Forcefor Intermediate Peclet Number Via von-Mises Coordinates
}

\author{
Mushtaq Ahmed \\ Department of Mathematics, University of Karachi, Karachi, Pakistan
}

Email address:

mushtaqku@yahoo.com, muahmed@uok.edu.pk

\section{To cite this article:}

Mushtaq Ahmed. On Two-Dimensional Variable Viscosity Fluid Motion with Body Forcefor Intermediate Peclet Number Via von-Mises Coordinates. International Journal of Fluid Mechanics \& Thermal Sciences. Vol. 5, No. 3, 2019, pp. 75-81.

doi: $10.11648 /$ j.ijfmts.20190503.13

Received: March 13, 2019; Accepted: July 26, 2019; Published: August 26, 2019

\begin{abstract}
This article uses von-Mises coordinates to present a class of new exact solutions of the system of partial differential equations for the plane steady motion of incompressible fluid of variable viscosity in presence of body forcefor moderate Peclet number. This communication applies successive transformation technique and characterizes streamlines through an equation relating a differentiable function $f(x)$ and a function of stream function. Considering the function of stream function satisfies a specific relation, the exact solutions for moderate Peclet number with body force are determined for given one component of the body force when $f(x)$ takes a specific value and when it is not. In both the cases, it shows an infinite set of streamlines, the velocity components, viscosity function, generalized energy function and temperature distribution for intermediate Peclet number in presence of body force. When $f(x)$ takes a specific value, a relation between viscosity and temperature function is observed.
\end{abstract}

Keywords: Variable Viscosity Fluids, Navier-Stokes Equations with Body Force, Martin's System, von-Mises Coordinates, Moderate Peclet Number

\section{Introduction}

For the motion of a variable viscosity fluidthe equation of conservation of mass, momentum and energy are known as a system of partial differential equations (PDE). The momentum equationsare Navier-Stokes equations (NSE). In Navier-Stokes equations, the product of mass and acceleration of fluid element are in left-hand side and body forces term in addition to surface forcein right-hand side. The non-dimensional equations for the steady motion of constant density and variable viscosity fluid in tensor notation are following:

$$
\frac{\partial v_{\alpha}}{\partial x_{\alpha}}=0
$$

$$
\begin{aligned}
v_{\beta} \frac{\partial v_{\alpha}}{\partial x_{\beta}}= & F_{\alpha}-\frac{\partial p}{\partial x_{\alpha}} \\
& +\frac{1}{R_{e}} \frac{\partial}{\partial x_{\beta}}\left\{\mu\left(\frac{\partial v_{\alpha}}{\partial x_{\beta}}+\frac{\partial v_{\beta}}{\partial x_{\alpha}}\right)\right\} \\
v_{\beta} \frac{\partial T}{\partial x_{\beta}}= & \frac{1}{R_{e} P_{r}} \frac{\partial}{\partial x_{\alpha}}\left(\frac{\partial T}{\partial x_{\alpha}}\right) \\
& +\frac{\mu E_{c}}{R_{e}} \frac{\partial v_{\alpha}}{\partial x_{\beta}}\left(\frac{\partial v_{\alpha}}{\partial x_{\beta}}+\frac{\partial v_{\beta}}{\partial x_{\alpha}}\right)
\end{aligned}
$$


where $F_{\alpha}\left(x_{\alpha}\right)$ is the body force per unit mass, $v_{\alpha}\left(x_{\alpha}\right)$ the fluid velocity, $p=p\left(x_{\alpha}\right)$ is pressure, the coefficients of viscosity $\mu>0$, the space coordinates $x_{\alpha}$ and $\alpha, \beta \in\{1,2,3\}$. The non-dimensionl quantities $E_{c}, R_{e}$ and $P_{r}$ are the Eckert number, the Reynolds number, the Prandtl number respectively.

For the two-dimensional Cartesian space case taking $\alpha, \beta \in\{1,2\}, x_{1}=x, x_{2}=y, v_{1}=u(x, y), v_{2}=v(x, y)$, $v_{3}=0, F_{1}=F_{1}(x, y), F_{2}=F_{2}(x, y), F_{3}=0$ in equations $(1-$ 3) one finds

$$
\begin{gathered}
u_{x}+v_{y}=0 \\
u u_{x}+v u_{y}=F_{1}-p_{x} \\
+\frac{1}{\mathrm{R}_{\mathrm{e}}}\left[\left(2 \mu u_{x}\right)_{x}+\left\{\mu\left(u_{y}+v_{x}\right)\right\}_{y}\right] \\
u v_{x}+v v_{y}=F_{2}-p_{y} \\
+\frac{1}{\mathrm{R}_{\mathrm{e}}}\left[\left(2 \mu v_{y}\right)_{y}+\left\{\mu\left(u_{y}+v_{x}\right)\right\}_{x}\right] \\
u\left(\frac{2}{a}\right)+v T_{y}=\frac{1}{P_{e^{\prime}}}\left(T_{x x}+T_{y y}\right) \\
+\frac{\mathrm{E}_{\mathrm{c}}}{\mathrm{R}_{\mathrm{e}}}\left[2 \mu\left(u_{x}^{2}+v_{y}^{2}\right)+\mu\left(u_{y}+v_{x}\right)^{2}\right]
\end{gathered}
$$

where $R_{e} P_{r}$ is the Peclet number $P_{e^{\prime}}$.

The solution of the equation (4) is a stream function $\psi(x, y)$ such that

$$
\frac{\partial \psi}{\partial y}=u \frac{\partial \psi}{\partial x}=-v
$$

The solutions of momentum and energy equations are there through dimension analysis methods and coordinates transformation techniques [1-6]. For solution of these equations when NSE includes body force some transformations technique are applied [7-10]. Further, solutions are there for very small and very large $P_{e^{\prime}}$ where as the solution with intermediate $P_{e^{\prime}}$ is challenging[11-14]. This communicationapplies successive transformation scheme to meet the challenge of moderate $P_{e^{\prime}}$. According to this scheme the basic non-dimensional flows equations with body force in Cartesian space $(x, y)$ are first transformed into Martin's coordinates $(\phi, \psi)$ then to von-Mises coordinates $(x, \psi)$. In Martin's coordinates, the curvilinear coordinates $(\phi, \psi)$ are such that the coordinate lines $\psi=$ const. are streamlines and the coordinate lines $\phi=$ constant are arbitrary [15]. Whereas in the von-Mises coordinates, the arbitrary coordinate lines of Martin's system is taken along the $x$-axis. Thus, the function $\phi=x$ and stream function $\psi$ of Martin's coordinates as independent variables instead of $y$ and $x$ [16]. Further, the characterization of the streamlines is through

$$
y-f(x)=\text { const. }
$$

where $f(x)$ is a differentiable function and $\psi=$ const. are the streamlines. Therefore, it is reasonable to take

$$
y=f(x)+v(\psi)
$$

with $v$ as a differentiable function of stream function $\psi$.

The paper is organized as follow: Section (2) shows transformation of basic equations into Martin's coordinates $(\phi, \psi)$. Section (3) retransforms equations from Martin's systemto von-Mises coordinates $(x, \psi)$. Section (4), calculates exact solutions in von-Mises coordinates. Last sectionpresents conclusions.

\section{Basic Equations to Martin's System}

Let us write the equations (5-7) to a convenient form, before transforming to Martin's coordinates, in terms of the vorticity function $\Omega$ and the total energy function $T_{x}$ defined by

$$
\begin{gathered}
\Omega=v_{x}-u_{y} \\
\mathrm{~L}=p+\frac{1}{2}\left(u^{2}+v^{2}\right)-\frac{1}{\mathrm{R}_{\mathrm{e}}} y_{\psi}
\end{gathered}
$$

and find

$$
\begin{gathered}
-v \Omega=F_{1}-L_{x}+\frac{1}{\mathrm{R}_{\mathrm{e}}} A_{y} \\
u \Omega=F_{2}-L_{y}-\frac{1}{R_{e}} B_{y}+\frac{1}{\mathrm{R}_{\mathrm{e}}} A_{x} \\
u T_{x}+v T_{y}=\frac{1}{P_{e^{\prime}}}\left(T_{x x}+T_{y y}\right) \\
+\frac{\mathrm{E}_{\mathrm{c}}}{\mathrm{R}_{\mathrm{e}}} \frac{1}{4 \mu}\left(B^{2}+4 A^{2}\right)
\end{gathered}
$$

where

$$
A=\mu\left(u_{y}+v_{x}\right) B=4 \mu u_{x}
$$

Let us consider the following allowable change of curvilinear coordinates $(\phi, \psi)$ through

$$
x=x(\phi, \psi), y=y(\phi, \psi)
$$


such that the Jacobian $J=\frac{\partial(x, y)}{\partial(\phi, \psi)}$ of the transformation is non-zero and finite. Let $\xi$ be the angle between the tangents to the streamlines $\psi=$ const . and the curves $\phi=$ const. at a common point $P(x, y)$, basic equations in Martin's system are following [17]

$$
\begin{aligned}
&-R_{e} \Omega J E= R_{e} J \sqrt{E}\left[\begin{array}{l}
-F\left(F_{1} \cos \xi+F_{2} \sin \xi\right) \\
+J\left(F_{1} \sin \xi-F_{2} \cos \xi\right)
\end{array}\right]+R_{e} J E L_{\psi}+ \\
& A_{\phi}\left(\left(F^{2}-J^{2}\right) \cos 2 \xi-2 F J \sin 2 \xi\right) \\
&\left.+E A_{\psi}(J \sin 2 \xi-F \cos 2 \xi)\right) \\
&-B_{\phi}\left(\frac{1}{2}\left(F^{2}-J^{2}\right) \sin 2 \xi+F J \cos 2 \xi\right) \\
&+E B_{\psi}\left(\frac{F}{2} \sin 2 \xi+J \cos ^{2} \xi\right), \\
& 0=R_{e} J \sqrt{E}\left[F_{1} \cos \xi+F_{2} \sin \xi\right] \\
&-R_{e} J L_{\phi}+E A_{\psi} \cos 2 \xi-A_{\phi}[F \cos 2 \xi-J \sin 2 \xi] \\
&+B_{\phi}\left(\frac{1}{2} F \sin 2 \xi-J \sin ^{2} \xi\right)-\frac{E B_{\psi}}{2} \sin 2 \xi,
\end{aligned}
$$

and

$$
\begin{gathered}
\frac{1}{\mathrm{~J} P_{e^{\prime}}}\left[\left(\frac{G T_{\phi}-F T_{\psi}}{J}\right)_{\phi}+\left(\frac{E T_{\psi}-F T_{\phi}}{J}\right)_{\psi}\right] \\
=-\frac{\mathrm{E}_{\mathrm{c}}}{\mathrm{R}_{\mathrm{e}}} \frac{1}{4 \mu}\left(B^{2}+4 A^{2}\right)+\frac{T_{\phi}}{J}
\end{gathered}
$$

where

$$
\begin{gathered}
E=x_{\phi}^{2}+y_{\phi}^{2}, \\
F=x_{\phi} x_{\psi}+y_{\phi} y_{\psi}, \\
G=\left(x_{\psi}\right)^{2}+\left(y_{\psi}\right)^{2}
\end{gathered}
$$

are the coefficients of first fundamental form and

$$
J= \pm \sqrt{E G-F^{2}}
$$

$$
\begin{gathered}
B(\phi, \psi)=\frac{4 \mu}{E J^{3}}\left[E_{\phi}(F \sin \xi+J \cos \xi)^{2}\right. \\
-2 E(F \sin \xi+J \cos \xi)\left(F_{\phi} \sin \xi+J_{\phi} \cos \alpha\right) \\
\left.+E^{2}\left(J_{\psi} \sin 2 \xi+G_{\phi} \sin ^{2} \xi\right)\right],
\end{gathered}
$$

$$
\begin{gathered}
A(\phi, \psi)=\mu\left[-\frac{(F \cos \xi-J \sin \xi)}{4 E^{2} J^{5}}\right. \\
\left\{E_{\phi}\left(2 E J^{3} \cos \xi+F \sqrt{E} \sin \xi\right)\right. \\
-4 E^{2} J^{2} J_{\phi} \cos \xi-2 E \sqrt{E} F_{\phi} \sin \xi \\
\left.+E \sqrt{E} E_{\psi} \sin \xi\right\} \\
+\frac{\cos \alpha}{2 J^{3}}\left\{E_{\psi}(F \sin \xi+J \cos \xi)\right. \\
\left.-2 E J_{\psi} \cos \xi-E G_{\phi} \sin \xi\right\} \\
+\frac{(F \sin \xi+J \cos \xi)}{2 E J^{3}}\left\{\left(J E_{\phi}-2 E J_{\phi}\right) \sin \xi\right. \\
\left.+\cos \xi\left[-F E_{\phi}+2 E F_{\phi}-E E_{\psi}\right]\right\} \\
-\frac{\sin \xi}{2 J^{3}}\left\{\left(E_{\psi}(J \sin \xi-F \cos \xi)\right.\right. \\
\left.\left.-2 E J_{\psi} \sin \xi+E G_{\phi} \cos \xi\right\}\right],
\end{gathered}
$$

and

$$
\begin{gathered}
\Omega=\frac{(F \sin \xi+J \cos \xi)}{2 E J^{3}}\left\{\left(J E_{\phi}-2 E J_{\phi}\right) \sin \xi\right. \\
\left.+\cos \xi\left[-F E_{\phi}+2 E F_{\phi}-E E_{\psi}\right]\right\} \\
-\frac{\sin \xi}{2 J^{3}}\left\{E_{\psi}(J \sin \xi-F \cos \xi)\right. \\
\left.\left.-2 E J_{\psi} \sin \xi+E G_{\phi} \cos \xi\right\}\right] \\
+\frac{(F \cos \xi-J \sin \xi)}{4 E^{2} J^{5}} \\
\left\{E_{\phi}\left(2 E J^{3} \cos \xi+F \sqrt{E} \sin \xi\right)\right. \\
-4 E^{2} J^{2} J_{\phi} \cos \xi-2 E \sqrt{E} F_{\phi} \sin \xi \\
\left.+E \sqrt{E} E_{\psi} \sin \xi\right\}
\end{gathered}
$$$$
-\left[\frac { \operatorname { c o s } \xi } { 2 J ^ { 3 } } \left\{E_{\psi}(F \sin \xi+J \cos \xi)-2 E J_{\psi} \cos \xi\right.\right.
$$$$
\left.\left.-E G_{\phi} \sin \xi\right\}\right]
$$

\section{Retransformation to von-Mises Coordinates}

Since $\phi=x$ is independent variable in von-Mises 
coordinates, thereforesetting

$$
\phi=x
$$

in equations (21-22), one have

$$
\begin{gathered}
E=1+\left[x f^{\prime}(x)\right]^{2} \\
F=J \sqrt{E-1} \\
G=x^{2} v^{\prime}(\psi)^{2} \\
J=x v^{\prime}(\psi)
\end{gathered}
$$

and the angle $\xi$ between the coordinate curvesis

$$
\cos \xi=\frac{1}{\sqrt{E}}
$$

Thus equations (18-20), equation (25) and equations (2324) are

$$
\begin{aligned}
& -R_{e} \Omega=-R_{e} J F_{2}+R_{e} L_{\psi}-J A_{x} \\
& +\sqrt{E-1} A_{\psi}+B_{\psi} \\
& 0=R_{e}\left(F_{1}+F_{2} \sqrt{E-1}\right)-R_{e} L_{x}+\frac{A_{\psi}(2-E)}{J} \\
& +A_{x} \sqrt{E-1}-\frac{\sqrt{E-1} B_{\psi}}{J} \\
& J T_{x x}-2 \sqrt{E-1} T_{v x} v^{\prime}+\frac{E}{J} T_{v v}\left(v^{\prime}\right)^{2} \\
& +\left(J_{x}-\frac{E_{\psi}}{2 \sqrt{E-1}}-P_{e^{\prime}}\right) T_{x} \\
& +\left(\frac{E_{\psi}}{J}-\frac{E_{x}}{2 \sqrt{E-1}}-\frac{E J_{\psi}}{J^{2}}+\frac{E}{J}\left(\frac{\nu^{\prime \prime}}{v^{\prime}}\right)\right) T_{\nu} \nu^{\prime} \\
& =-\frac{J E_{c} P_{r}}{4 \mu}\left(B^{2}+4 A^{2}\right) \\
& \Omega=\frac{1}{v^{\prime}(\psi)}\left[\begin{array}{l}
\left\{\frac{f^{\prime}(x)}{x}+f^{\prime \prime}(x)\right\} \\
+\left\{\frac{1}{x^{2}}+\left[f^{\prime}(x)\right]^{2}\right\}\left(\frac{v^{\prime \prime}(\psi)}{\left\{v^{\prime}(\psi)\right\}^{2}}\right)
\end{array}\right]
\end{aligned}
$$

where

$$
A(x, \psi)=\frac{\mu}{J}\left[\begin{array}{l}
\frac{-2 J_{x} \sqrt{E-1}}{J}+\frac{E_{x}}{2 \sqrt{E-1}} \\
-\frac{(2-E) J_{\psi}}{J^{2}}
\end{array}\right]
$$

$$
B(x, \psi)=4 \mu \frac{1}{J^{3}}\left[-J J_{x}+\sqrt{E-1} J_{\psi}\right]
$$

and $q$ the magnitude of $\mathrm{q}=(u, v)$ is

$$
q=\frac{\sqrt{E}}{J}
$$

\section{Exact Solutions in von-Mises Coordinates}

Follow [1-3], the condition $L_{x \psi}=L_{\psi x}$ on equations (2930) provides

$$
\begin{aligned}
& x v^{\prime} A_{x x}-2 x f^{\prime} A_{x \psi}-\frac{\left[1-x^{2}\left(f^{\prime}\right)^{2}\right]}{x v^{\prime}} A_{\psi \psi} \\
+ & v^{\prime} A_{x}-A_{\psi}\left(f^{\prime}+x f^{\prime \prime}\right)-\left\{B_{x}-\frac{f^{\prime} B_{\psi}}{v^{\prime}}\right\}_{\psi} \\
= & R_{e} \Omega_{x}+R_{e}\left(F_{1}+F_{2} x f^{\prime}\right)_{\psi}-R_{e}\left(x v^{\prime} F_{2}\right)_{x}
\end{aligned}
$$

The solution of equation (39) is expected to lead to the exact solution therefore let us simplify $\Omega$ involved in the right-hand side of it through

$$
v^{\prime \prime}=d v^{\prime 2}
$$

where $d$ is constant. The case for $d=0$ is considered separately. For $d \neq 0$ equation (40)gives

$$
v=\frac{1}{d} \ln \left[\frac{-1}{d\left(k_{1} \psi+k_{2}\right)}\right]
$$

where $k_{1}$ and $k_{2}$ are constants. Equations (35-37) using (40) implies

$$
\begin{gathered}
\Omega=\left(\frac{1}{v^{\prime}}\right)\left[\frac{N^{\prime}}{x}+\frac{c\left(1+N^{2}\right)}{x^{2}}\right], \\
A=\frac{\mu}{x^{2} v^{\prime}}\left[x N^{\prime}-2 N-d\left(1-N^{2}\right)\right]
\end{gathered}
$$

and

$$
B=\frac{4 \mu}{x^{2} v^{\prime}}[-1+d N]
$$

Where

$$
N(x)=x f^{\prime}(x)
$$

Let us attempt solutions of equation (39) by eliminating $\mu$ from equations (43-44) and introducing function $Y(x)$ 
through

$$
A=Y(x) B
$$

where

$$
Y(x)=\frac{x N^{\prime}-2 N-d\left(1-N^{2}\right)}{4(-1+d N)} \neq 0
$$

Thus, it becomes

$$
\begin{gathered}
x Y B_{x x}-(1+2 N Y) B_{v x}+B_{v v}\left(\frac{N-\left(1-N^{2}\right) Y}{x}\right) \\
+B_{v}\left[-2 N Y^{\prime}-Y N^{\prime}\right]+B_{x}\left(2 x Y^{\prime}+Y\right) \\
+B\left(x Y^{\prime \prime}+Y^{\prime}\right)=R_{e}\left(\frac{1}{v^{\prime 2}}\right)\left[\frac{N^{\prime}}{x}+\frac{d\left(1+N^{2}\right)}{x^{2}}\right] \\
+R_{e}\left(F_{1}+N F_{2}\right)_{v}-R_{e}\left(x F_{2}\right)_{x}
\end{gathered}
$$

The use of (41) and (46) in the equation (34)involves factor $\left(1-\frac{P_{e^{\prime}}}{v^{\prime}}\right)$ therefore, this guides to searchthe function $B$ of the type

$$
B(x, \psi)=\left(1-\frac{P_{e^{\prime}}}{v^{\prime}}\right) R(x)
$$

where the function $R(x)$ is to be determined. Equation (48) on utilizing equation (49)gives

$$
\begin{gathered}
\left(1-\frac{P_{e^{\prime}}}{v^{\prime}}\right)\left[x Y R^{\prime \prime}+R^{\prime}\left(2 x Y^{\prime}+Y\right)+R\left(x Y^{\prime \prime}+Y^{\prime}\right)\right] \\
+\frac{d P_{e^{\prime}}}{v^{\prime}}\left[-(1+2 N Y) R^{\prime}-R\left(\frac{d\left\{N-\left(1-N^{2}\right) Y\right\}}{x}\right)\right] \\
\left.\left.=R_{e}\left(\frac{1}{v^{\prime 2}}\right)\left[\frac{N^{\prime}}{x}+\frac{d\left(1+N^{\prime}\right)}{x^{2}}\right]^{\prime}\right)\right]+R_{e}\left(F_{1}\right)_{v} \\
+R_{e} N\left(F_{2}\right)_{v}-R_{e}\left(x F_{2}\right)_{x}
\end{gathered}
$$

The search for the appropriate form of $F_{1}$ and $F_{2}$ providing the solution of equations (32-34) leads to $F_{2}(x, \psi)=F_{2}(x)$ as a solution of the following differential equation

$$
R_{e}\left(\operatorname{ax} F_{2}\right)_{x}=-\left[x(Y R)^{\prime}\right]^{\prime}
$$

or

$$
R_{e} F_{2}=-(Y R)^{\prime}+\frac{h_{1}}{x}
$$

where $h_{1}$ is constant.

The equation (50) and (52)implies

$$
\begin{gathered}
R_{e} F_{1}=\left(\frac{P_{e^{\prime}}}{c K_{1}}\right)\left[x(Y R)^{\prime}\right] e^{-d v} \\
\left.+\frac{P_{e^{\prime}} e^{-d v}}{K_{1}}\left[\begin{array}{l}
-(1+2 N Y) R^{\prime} \\
-R\left(\frac{d\left\{N-\left(1-N^{2}\right) Y\right\}}{x}\right) \\
+2 N Y^{\prime}+Y N^{\prime}
\end{array}\right)\right] \\
+\left(\frac{R_{e} e^{-2 d v}}{2 d K_{1}^{2}}\right)\left[\frac{N^{\prime}}{x}+\frac{d\left(1+N^{2}\right)}{x^{2}}\right]+H(x)
\end{gathered}
$$

Substitution of equations (52-53) in equations (32-33) provides the function $L$

$$
\begin{gathered}
R_{e} L=v h_{1}+\left(\frac{R_{e} e^{-2 d v}}{2 d K_{1}^{2}}\right)\left[\frac{N^{\prime}}{x}+\frac{d\left(1+N^{2}\right)}{x^{2}}\right] \\
+\frac{P_{e^{\prime}} e^{-d v}}{K_{1}}\left[\frac{x(Y R)^{\prime}}{d}+(N Y+1) R\right] \\
+\int\left(\frac{N h_{2}}{x}+2 N(Y R)^{\prime}+H(x)\right) d x+h_{2}
\end{gathered}
$$

where $h_{2}$ is constant and thus equation(43) or equation (44) provides

$$
\mu=-\frac{v^{\prime} x^{2}}{4(-1+d N)}\left(1-\frac{P_{e^{\prime}}}{v^{\prime}}\right) R(x)
$$

The equation (34) on using (27), (40), (46), (49) and (55) becomes

$$
\begin{gathered}
\left(x v^{\prime}\right) T_{x x}-2 N T_{v x} v^{\prime}+\frac{\left(1+N^{2}\right)}{x} T_{v v}\left(v^{\prime}\right) \\
+\left(v^{\prime}-P_{e^{\prime}}\right) T_{r}-N^{\prime} T_{v} v^{\prime} \\
=-\frac{E_{c} P_{r}(-1+d N)}{x}\left(1+4 Y^{2}\right)\left(1-\frac{P_{e^{\prime}}}{v^{\prime}}\right) R(x)
\end{gathered}
$$

The right-hand side of equation (56) suggestssearching for its solution of the type

$$
T(x, v)=\frac{K(x)}{v^{\prime}}
$$

where $K(x)$ is unknown function to be determined. Utilization of equation (57) in equation (56), provides 


$$
\begin{array}{r}
x K^{\prime \prime}+2 d N K^{\prime}+\frac{d^{2}\left(1+N^{2}\right) K}{x} \\
+\left(1-\frac{P_{e^{\prime}}}{v^{\prime}}\right) K^{\prime}+d K N^{\prime} \\
=-\frac{E_{c} P_{r}(-1+d N)}{x}\left(1+4 Y^{2}\right)\left(1-\frac{P_{e^{\prime}}}{v^{\prime}}\right) R(x)
\end{array}
$$

Comparing the coefficient of $\left(1-\frac{P_{e^{\prime}}}{v^{\prime}}\right)$ on both side of equation (58), one finds

$$
R(x)=-\frac{x K^{\prime}}{E_{c} P_{r}(-1+d N)\left(1+4 Y^{2}\right)}
$$

and

$$
x^{2} K^{\prime \prime}+2 d x N K^{\prime}+K\left[d^{2}\left(1+N^{2}\right)+d x N^{\prime}\right]=0
$$

Equation (59) and (60) are coupled equations, the function $K(x)$ from equation (60) will lead to $R(x)$ from equation (59) and the solution of equation (60) can be found for a choice of $f(x)$. For example considering $f(x)=m_{1} \ln x+m_{2}$ it reduces to Cauchy-Euler equation, when $f(x)=\frac{1}{d} \ln \cos \left(m_{3}-d \ln x\right)+m_{4}$, reduction of order method is applicable and when $f(x)=\frac{x^{2}}{2 d}-d \ln x$, the equation is solvable by transforming to normal form. For other $f(x)$, the solution of variable coefficient differential equation (60) is easy to find from computer algebra system (CAS) software. This leads to $T$ from equation (57), $\mu$ from equation (55), $p$ from (12) using equation (54) and $\mathrm{q}=(u, v)$ from equation (8) for $F_{1}$ and $F_{2}$ from equations (52-53) for intermediate Peclet number.

When $Y(x)=0$, the equation (47) implies

$$
x N^{\prime}-2 N-d\left(1-N^{2}\right)=0
$$

Since $d \neq 0$ therefore solution of equation (61) for $d=1$ and $d=-1$ is presented as an example and likewise one can find for $d>0$ or $d<0$. When $d=1$ equation (61) provides

$$
f(x)=c_{2}+\ln x+\ln \left[\cosh \left(\sqrt{2}\left(c_{1}-\ln x\right)\right)\right]
$$

and for $d=-1$ it provides

$$
f(x)=c_{2}-\ln x-\ln \left[\cosh \left(\sqrt{2}\left(c_{1}+\ln x\right)\right)\right]
$$

For both the equations(62-63), the equation (43) gives

$$
A=0
$$

Using (64) in (39), we get

$$
\begin{gathered}
-\left\{B_{x}-\frac{f^{\prime} B_{\psi}}{v^{\prime}}\right\}_{\psi}=R_{e} \Omega_{x} \\
+R_{e}\left(F_{1}+F_{2} x f^{\prime}\right)_{\psi}-R_{e}\left(x v^{\prime} F_{2}\right)_{x}
\end{gathered}
$$

Our search for the appropriate form of $F_{1}$ and $F_{2}$ providing the solution of equations (32-34) leads to $F_{2}(x, \psi)=F_{2}(x)$ as a solution of the following differential equation

$$
R_{e}\left(x v^{\prime} F_{2}\right)_{x}=R_{e} \Omega_{x}
$$

or

$$
R_{e}\left(x v^{\prime} F_{2}\right)=R_{e} \Omega+G(\psi)
$$

where the function of integration is $G(\psi)$. Utilizing (67) in (65), one finds

$$
R_{e} F_{1}=-R_{e} F_{2} x f^{\prime}-\left\{B_{x}-\frac{f^{\prime} B_{\psi}}{v^{\prime}}\right\}+H_{1}(x)
$$

where $H_{1}(x)$ is a function of integration. Solving (32-33), using equations (67-68), we have

$$
R_{e} L=-B-\int G(\psi) d \psi+\int H_{1}(x) d x+h_{3}
$$

where $h_{3}$ is constant.

In light of equation (64), the equation (34)becomes

$$
\begin{gathered}
x T_{x x}-2 N T_{v x}+\frac{\left(1+N^{2}\right)}{x} T_{v v}+\left(1-\frac{P_{e^{\prime}}}{v^{\prime}}\right) T_{x} \\
-N^{\prime} T_{v}=-\frac{x E_{c} P_{r} B^{2}}{4 \mu}
\end{gathered}
$$

The function $B$ from equation (70) in equation (44) gives a relation between viscosity andtemperature function

$$
\mu=\frac{-x^{3}\left(v^{\prime}\right)^{2}}{4 E_{c} P_{r}(d N-1)^{2}}\left[\begin{array}{l}
x T_{x x}-2 N T_{v x} \\
+\frac{\left(1+N^{2}\right)}{x} T_{v v} \\
+\left(1-\frac{P_{e^{\prime}}}{v^{\prime}}\right) T_{x} \\
-N^{\prime} T_{v}
\end{array}\right]
$$

The streamline patterns can be drawn using CAS software to observe the effect of various parameters for $d$ either $+v e$ or $-v e$. 


\section{Conclusion}

This communication finds a class of new exact solutions of the equations governing the two-dimensional steady motion with moderate Peclet number of incompressible fluid of variable viscosity in presence of body force in von-Mises coordinates. The characteristic equation for the streamlinesis the equation $y=f(x)+\frac{1}{d} \ln \left[\frac{-1}{d\left(k_{1} \psi+k_{2}\right)}\right]$ where the differentiable function $f(x)$ is either $f(x)=c_{2}+\ln \left[x \cosh \left(\sqrt{2}\left(c_{1}-\ln x\right)\right)\right]$ or $f(x)=c_{2}-\ln \left[x \cosh \left(\sqrt{2}\left(c_{1}+\ln x\right)\right)\right]$, $\psi$ is the stream function and the constants $d$ is either $+v e$ or $-v e$. The pressure $p$ and velocity components for given the component of body force $F_{1}$ or $F_{2}$ are found when Peclet number is moderate and a relation between viscosity $\mu$ and temperature function $T$ is observed. For other $f(x)$ in streamlines equation, temperature, viscosity, pressure and velocity components for given component of body force are found for moderate Peclet number. It shows that in both the cases an infinite set of velocity components, viscosity function, generalized energy function and temperature distribution for intermediatePeclet number in presence of body force can be constructed and graph of streamlines using CAS software can be drawn to observe the streamline patterns.

\section{References}

[1] Mushtaq A., On Some Thermally Conducting Fluids: Ph.D Thesis, Department of Mathematics, University of Karachi, Pakistan, 2016.

[2] Chandna, O. P., Oku-Ukpong E. O.; Flows for chosen vorticity functions-Exactsolutions of the Navier-Stokes Equations: International Journal of Applied Mathematics and Mathematical Sciences, 17 (1) (1994) 155-164.

[3] Naeem, R. K.; Srfaraz, A. N.; Study of steady plane flows of an incompressible fluid of variable viscosity using Martin's System: Journal of Applied Mechanics and Engineering, 1996, 1 (1), 397-433.

[4] Naeem, R. K.; Steady plane flows of an incompressible fluid of variable viscosity via Hodograph transformation method: Karachi University Journal of Sciences, 2003, 3 (1), 73-89.

[5] Naeem, R. K.; On plane flows of an incompressible fluid of variable viscosity: Quarterly Science Vision, 2007, 12 (1), 125-131.

[6] Naeem, R. K.; Mushtaq A.; A class of exact solutions to the fundamental quations for plane steady ncompressible and variable viscosity fluid in the bsence of body force: International Journal of Basic and Applied Sciences, 2015, 4
(4), 429-465. www.sciencepubco.com/index.php/IJBAS, doi: 10.14419/ijbas.v4i4.5064.

[7] Mushtaq A.; Naeem R. K.; S. Anwer Ali; A class of new exact solutions of Navier-Stokes equations with body force for viscous incompressible fluid,: International Journal of Applied Mathematical Research, 2018, 7 (1), 22-26. www.sciencepubco.com/index.php/IJAMR, doi: 10.14419/ijamr.v7i1.8836.

[8] Mushtaq Ahmed, Waseem Ahmed Khan: A Class of New Exact Solutions of the System ofPDEfor the plane motion of viscous incompressible fluids in the presence of body force,:International Journal of Applied Mathematical Research, 2018, 72 (2), 48.www.sciencepubco.com/index.php/IJAMR, doi: 10.14419/ijamr.v7i2.9694.

[9] Mushtaq Ahmed, Waseem Ahmed Khan, S. M. Shad Ahsen:A Class of Exact Solutions of Equations for Plane Steady Motion of Incompressible Fluids of Variable viscosity in presence of Body Force,:International Journal of Applied Mathematical Research, 2018, 7 (3), 77 81.www.sciencepubco.com/index.php/IJAMR, doi: 10.14419/ijamr.v7i2.12326.

[10] Mushtaq Ahmed, A Class of New Exact Solution of equations for Motion of Variable Viscosity Fluid In presence of Body Force with Moderate Peclet number, International Journal of Fluid Mechanics and Thermal Sciences, 2018, 4 (3) 2733www.sciencepublishingdroup.com/j/ijfmts doi: 10.11648/j.ijfmts.20180403.11.

[11] D. L. R. Oliver \& K. J. De Witt, High Peclet number heat transfer from adroplet suspended in an electric field: Interior problem, Int. J. Heat Mass Transfer, vol. 36: 3153-3155, 1993.

[12] B. Abramzon and C. Elata, Numerical analysis of unsteady conjugate heattransfer between a single spherical particle and surrounding flow at intermediate Reynolds and Peclet numbers, 2nd Int. Conf. on numerical methods in Thermal problems, Venice, pp. 1145-1153, 1981.

[13] Z. G. Fenz, E. E. Michaelides, Unsteady mass transport from a sphere immersedin a porous medium at finite Peclet numbers, Int. J. Heat Mass Transfer 42:3529-3531, 1999.

[14] Fayerweather Carl, Heat Transfer From a Droplet at Moderate Peclet Numbers with heat Generation. Ph.D. Thesis, U of Toledo, May 2007.

[15] Martin, M. H.; The flow of a viscous fluid I: Archive for Rational Mechanics and Analysis, 1971, 41 (4), 266-286.

[16] Daniel Zwillinger; Handbook of differential equations; Academic Press, Inc. (1989).

[17] Mushtaq Ahmed, A Class of Exact Solutions for a Variable Viscosity Flowwith Body Force for Moderate Peclet Number Via Von-Mises Coordinates, Fluid Mechanics, 2019, 5 (1), 1525, www.sciencepublishinggroup.com $/ \mathrm{j} / \mathrm{fm}$ doi: 10.11648/j.fm.20190501.13. 